\title{
Asociación entre la morfología alar y el uso del hábitat en seis especies de palomas (Columbidae) neotropicales
}

\author{
Diego Ocampo ${ }^{1,2 *}$, Angélica Alvarado ${ }^{3}$, María José Álvarez ${ }^{3}$, J. Adrián Ríos-Acuña ${ }^{3}$, \\ Gilbert Barrantes ${ }^{2,3} \&$ Luis Sandoval ${ }^{2,3}$ \\ 1. Department of Biology, University of Miami, Coral Gables, 33146 USA; ocampov.diego@gmail.com \\ 2. Museo de Zoología, Escuela de Biología, Universidad de Costa Rica, CP-11501-2060, San Pedro, San José, Costa \\ Rica; gilbert.barrantes@gmail.com, biosandoval@gmail.com \\ 3. Escuela de Biología, Universidad de Costa Rica, CP-11501-2060, San Pedro, San José, Costa Rica; \\ 92alvarado@gmail.com, chepima95@gmail.com, joseht.ad.rios@gmail.com \\ * Correspondencia
}

Recibido 06-X-2017. Corregido 18-I-2019. Aceptado 17-I-2019.

\begin{abstract}
Relationship between wing morphology and habitat use in six species of Neotropical doves (Columbidae). Biomechanics, behavior, and natural history influence wing dimension and shape. Wing design often correlates with features of the habitat in which each species is found. Doves and pigeons (Columbidae family) range from long-distance fliers (e.g., canopy and open area species) to very short-distance fliers (e.g., species adapted to dense understory forests) and such variation makes this group fit to test the association between flying habits and wing morphology. Our objective in this study is to determine whether the wing morphology (shape and dimensions) of six dove species is associated to their flying capability. We predict that the long-distance fliers Patagioenas flavirostris and P. nigrirostris will have long and sharp wings; while the very short-distance flier Geotrygon montana will have broad and rounded wings. Other species (e.g., Leptotila verreauxi, L. cassini and Zenaida asiatica) whose flying capability fits in between these two will have wings with intermediate morphological features. We measured the wing disc loading, shape ratio, the ratio of mean to maximum wing chord, relative wing length, and wing area for each species. We conducted a discriminant function analysis to compare which variables explain better the differences in wing morphology across the six species, and used a binomial test to evaluate the power of the model. The model correctly classified $57 \%$ of individuals within their own species. The flying capability is associated with the wing morphology of the six Columbidae species; with a wing design for long-distance fliers in P. flavirostris and P. nigrirostris, wing design for maneuvering in dense habitats in G. montana, and wings with an intermediate design in L. verreauxi, L. cassini and Z. asiatica.
\end{abstract}

Key words: Aerodynamic parameters; Flight behavior; Habitat effect; Pigeons; Wing shape.

Ocampo, D., Alvarado, A., Álvarez, M. J., J. Ríos-Acuña, A., Barrantes, G. \& Sandoval, L. (2019). Asociación entre la morfología alar y el uso del hábitat en seis especies de palomas (Columbidae) neotropicales. Revista de Biología Tropical, 67(2) Suplemento, S315-S325.

La evolución de la forma y dimensión de las alas en las aves está influenciada por la interacción entre factores físicos (peso corporal, forma del cuerpo y biomecánica de las alas), ecológicos (hábitat), así como la historia natural de cada especie (tipo de alimentación y cortejo) (Savile, 1957; Arizaga, Campos, \&
Alonso, 2006; Corvidae, Bierregaard, \& Peters, 2006). Por ejemplo, las alas cortas, anchas, y redondeadas se han asociado a vuelos potentes de alta maniobrabilidad, pero de poca duración, por lo que están presentes en aves que vuelan poco y caminan mucho (ej., galliformes, rálidos, o tamnofílidos de sotobosque) (Kirmse, 
1998; Lockwood, Swaddle, \& Rayner, 1998). Por otro lado, las alas largas y puntiagudas están presentes en especies que vuelan largas distancias a alta velocidad y por extensos periodos de tiempo (ej., vencejos, halcones, o carádridos) (Norberg, 1995; Corvidae et al., 2006).

Debido a que el vuelo es la principal forma de locomoción para la mayoría de las especies de aves y que representa un alto costo energético respecto a otras formas de desplazamiento (Videler, 2005), es intuitivo pensar que las formas de las alas, y la anatomía muscular y esquelética en cada especie debe estar adaptada al ambiente en el que habitan y a sus requerimientos diarios (Norberg, 1981; Dial, 1992). Por lo tanto, se espera que especies relacionadas filogenéticamente, que utilizan diferentes hábitats (ej., zonas abiertas vs sotobosques densos), o que presentan diferentes estrategias de forrajeo (ej., caza de presas al vuelo vs búsqueda de semillas en el suelo), presenten diferente morfología alar y adaptaciones musculares y esqueléticas asociadas al ambiente (Corvidae et al., 2006). Mientras que especies no relacionadas filogenéticamente, pero que utilizan el mismo hábitat (ej., sotobosques densos), o que presentan la misma estrategia de forrajeo (ej., cazan presas al vuelo), debieran presentar similar morfología alar y adaptaciones corporales y esqueléticas (Corvidae et al., 2006).

Nuestro objetivo es determinar si la capacidad de vuelo (ej., larga vs corta distancia) y el hábitat principal por el que se desplazan (ej., zonas abiertas vs. hábitats densos) se asocian con la morfología alar en seis especies de cuatro géneros de palomas. Seleccionamos estas seis especies porque presentan una alta variación en el tiempo y distancia de vuelo y su relación filogenética cercana, lo que reduce el sesgo filogenético. El grupo de palomas incluye especies que habitan principalmente en el dosel y que se desplazan principalmente volando largas distancias (Patagioenas flavirostris y $P$. nigrirostris); especies que utilizan principalmente el subdosel de bosques secundarios y zonas abiertas (Zenaida asiatica); especies que utilizan sotobosques abiertos y de plantaciones forestales y se desplazan caminando y volando (Leptotila verreauxi y L. cassini); y especies que habitan en sotobosques densos y que se desplazan poco, principalmente caminando (Geotrygon montana) (Stiles, \& Skutch, 1989; Skutch, 1991; Gibbs, Bames, \& Cox, 2001; Lapiedra, Sol, Carranza, \& Beaulieu, 2013). Predecimos que las especies del género Patagioneas, que se desplazan principalmente volando y en el dosel, tienen alas relativamente más largas que anchas y más puntiagudas. Este tipo de morfología alar disminuye el costo energético del vuelo en zonas abiertas (Savile, 1957). Geotrygon montana que se desplazan principalmente caminando y en el interior de sotobosques densos, tendrían alas relativamente más anchas que largas y poco puntiagudas. Esta forma favorece una mayor maniobrabilidad en ambientes con vegetación densa (Savile, 1957). Especies con una capacidad de vuelo intermedia (ej., L. verreauxi, L. cassini y $Z$. asiatica) presentarían alas con características también intermedias en su morfología alar.

\section{MATERIALES Y MÉTODOS}

Obtuvimos las medidas de las alas y el peso de los de los individuos de seis especies y cuatro géneros (Cuadro 1) de dos fuentes: aves vivas medidas en el campo, e individuos depositados en el Museo de Zoología, Universidad de Costa Rica (MZUCR) y Museo Nacional de Costa Rica (MNCR). Las medidas de los individuos depositados en museos se obtuvieron previo a la preservación de los mismos (frescos). Debido a esta condición, no esperamos que haya efecto en la variación de la forma alar asociada a la fuente de los datos.

Clasificamos las especies analizadas según el uso del hábitat predominante y la proporción de tiempo que se desplazan volando, basados en los reportes de la literatura (Stiles \& Skutch 1989; Baptista, Trail, \& Horblit, 1997; Gibbs et al. 2001) y nuestras observaciones en campo para cada especie. La clasificación se hizo tomando en cuenta tres categorías: el tipo de hábitat más frecuentemente usado por cada especie $($ dosel $=5$, dosel-subdosel $=4$, subdosel $=3$, sotobosque abierto $=2$, y sotobosque 
CUADRO 1

Número de especímenes de cada una de las especies analizadas por sexo (? = individuos de sexo desconocido), y las características del uso de hábitat y vuelo reportado en la literatura* utilizados para asignar los valores de índice de vuelo

TABLE 1

Abundance of each species per sex $(?=$ unknown sex $)$ used in the study, and the characteristics of the habitat and flight behavior reported in literature* used to estimate the flight index

\begin{tabular}{lcccclllc}
\multicolumn{1}{c}{ Especie } & Machos & Hembras & $?$ & Total & Hábitat & $\begin{array}{c}\text { Zonas } \\
\text { abiertas }\end{array}$ & $\begin{array}{c}\text { Desplazamiento } \\
\text { Índice } \\
\text { de vuelo }\end{array}$ \\
Geotrygon montana & 2 & 5 & 5 & 12 & sotobosque denso & poco & caminar & 3 \\
Leptotila cassini & 2 & 5 & 7 & 14 & sotobosque denso & intermedio & caminar-volar & 5 \\
L. verreauxi & 7 & 2 & 21 & 30 & sotobosque abierto & intermedio & caminar-volar & 6 \\
Zenaida asiatica & 3 & 1 & 24 & 28 & subdosel & común & volar-caminar & 9 \\
Patagioenas nigrirostris & 3 & 1 & 6 & 10 & dosel-subdosel & intermedio & vuelo & 10 \\
P. flavirostris & 1 & 1 & 15 & 17 & dosel & común & vuelo & 12 \\
\hline
\end{tabular}

Los valores de cada categoría dentro de Hábitat, Zonas abiertas y Desplazamiento, se encuentran explicadas con detalle en los materiales y métodos.

*Literatura utilizada para extraer la información de hábitat y vuelo: Stiles \& Skutch, 1989; Baptista et al., 1997; Gibbs et al., 2001.

Values of each category of Habitat, Open Areas, and Movement are explained with detail in methods.

*Literature used to obtain the habitat and flight behavior: Stiles \& Skutch, 1989; Baptista et al., 1997; Gibbs et al., 2001.

denso $=1)$, el uso de zonas abiertas para desplazarse $($ común $=3$, intermedio $=2$, y poco $=$ 1), y el modo de desplazamiento más común (vuelo $=4$, vuelo-caminar $=3$, caminar-vuelo $=2$, y caminar $=1$ ). A cada especie se le asignó un único valor por categoría, la sumatoria de los valores de las tres categorías, nos dan un estimado de la capacidad de vuelo y el hábitat por el que se desplazan (índice de vuelo). Especies con valores del índice de vuelo de 3 a 5 indica que habitan sotobosques densos y utilizan la caminata como principal medio de locomoción, por lo que se espera que presenten alas relativamente anchas y redondeadas. Especies con valores del índice de vuelo de 10 a 12 indica que hacen uso de zonas abiertas y utilizan el vuelo como principal medio de locomoción, por lo tanto, esperamos que estas especies presenten alas relativamente largas y puntiagudas. Especies con valores del índice de vuelo de 6 a 9 utilizan proporciones similares vuelo y caminar como medio de locomoción, por lo tanto, se espera que presenten valores intermedios de la morfología alar (Cuadro 1).
Pesamos cada individuo (g) utilizando una balanza mecánica marca PESOLA de $\pm 1 \mathrm{~g}, \mathrm{y}$ dibujamos el contorno del ala abierta, en posición natural de vuelo, sobre un papel (Fig. 1). De cada ala dibujada (Fig. 1) medimos (1) el largo del ala (LA): la distancia $(\mathrm{cm})$ desde la punta al extremo anterior de la base del ala; (2) el ancho del ala (WA): la distancia máxima $(\mathrm{cm})$ perpendicular a LA entre el borde de ataque y de salida del ala; y (3) el área alar (AA): área total del ala para lo cual usamos el programa ImageJ (Schneider, Rasband, \& Eliceiri, 2012). Con estas características de las alas estimamos cuatro parámetros aerodinámicos (Stiles, Altshuler, \& Dudley, 2005; Pennycuick, 2008), y seguimos la nomenclatura propuesta para dichos parámetros en aves por Stiles et al. (2005); (1) la carga alar (wing loading): masa en $\mathrm{g} / 2 * \mathrm{AA}$, la cual indica el impulso de presión requerido para mantener el vuelo estacionario; (2) la proporción de forma (shape ratio): LA/ WA, que indica si el ala tiende a ser cuadrada (igual a 1) o rectangular; (3) la conicidad alar (wing taper): $\left\{\left[2 *(\mathrm{LA})^{2} / \mathrm{AA}\right] /(2 *\right.$ proporción de forma) $\}-1$, que indica que tan puntiaguda 
A

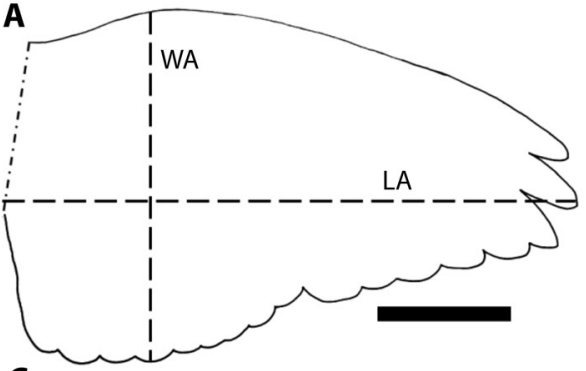

C

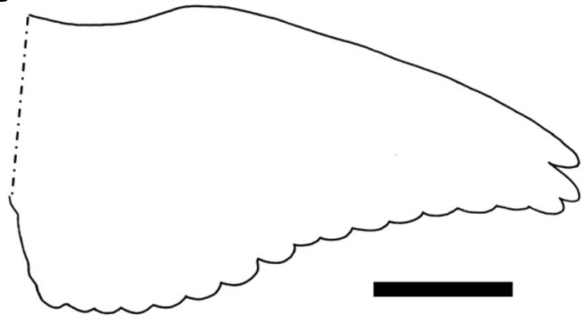

E

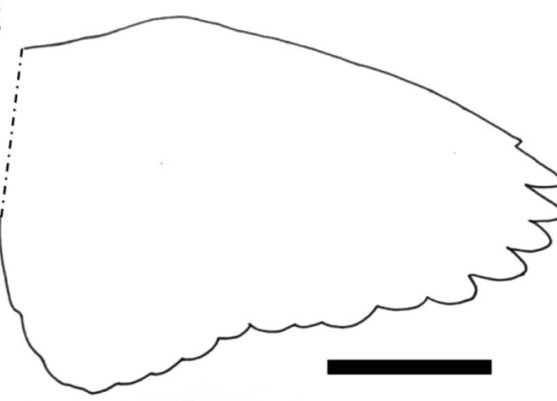

B

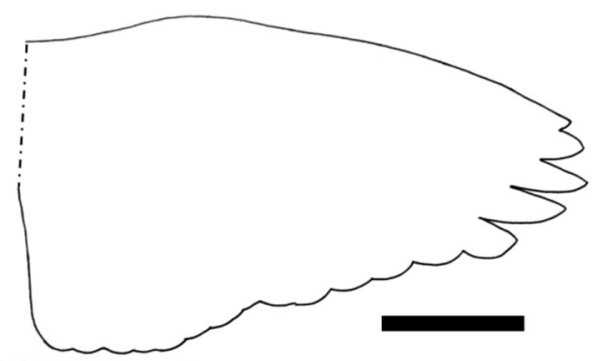

D

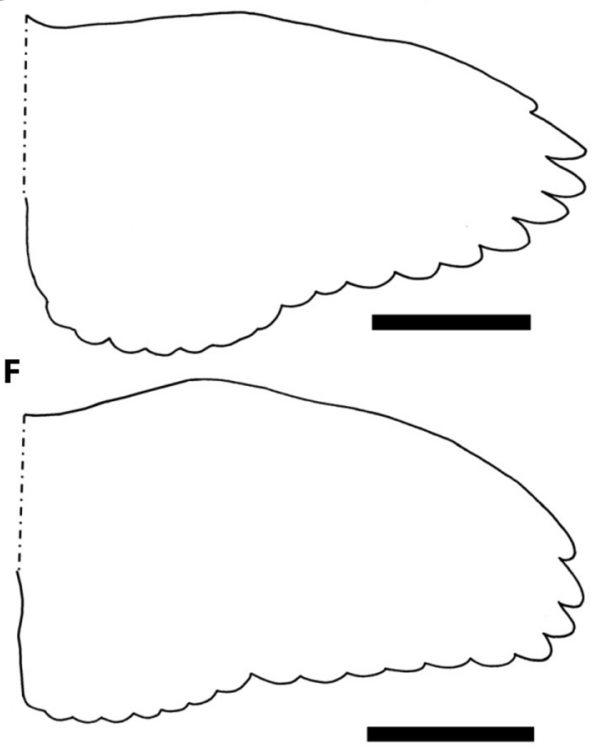

Fig. 1. Ejemplos del contorno del ala de cada género de paloma. (A) Patagioenas flavirostris, (B) P. nigrirostris, (C) Zenaida asiatica, (D) Leptotila verreauxi, (E) L. cassini, y (F) Geotrygon montana. La barra negra representa $5 \mathrm{~cm}$. LA: distancia $(\mathrm{cm})$ entre la punta y la base del ala extendida en la posición natural de vuelo. WA: distancia máxima $(\mathrm{cm})$ perpendicular a LA entre el borde de ataque y de salida del ala en la posición natural de vuelo.

Fig. 1. Examples of wing contour of each dove genera. (A) Patagioenas flavirostris, (B) P. nigrirostris, (C) Zenaida asiatica, (D) Leptotila verreauxi, (E) L. cassini, y (F) Geotrygon montana. The black line is $5 \mathrm{~cm}$. LA: distance (cm) between wing tip and wing base in flight position. WA: maximum distance $(\mathrm{cm})$ perpendicular to LA between the front and bottom part of the wing in flight position.

es el ala (Stiles et al., 2005); y (4) el largo del ala relativo (relative length): LA/ (masa en $\mathrm{g})^{1 / 3}$, que indica la longitud del ala una vez corregido por el tamaño de cada individuo.

Análisis estadísticos: Debido a que algunas especies están más relacionadas entre sí que otras (ej., las dos especies de Patagioenas y las dos especies de Leptotila), decidimos analizar si la variación de los cuatro parámetros aerodinámicos (carga alar, proporción de forma, conicidad alar, y largo relativo) y el área alar, que son las variables dependientes, puede ser explicados por las relaciones filogenéticas entre las especies. Para esto, estimamos el valor del parámetro lambda $(\lambda)$ de la correlación entre las variables en el árbol de consenso filogenético, construido utilizando la regla de la mayoría del $50 \%$ con el programa Mesquite 3.04 (Maddison \& Maddison, 2015) y derivado de 10000 árboles, los cuales incluían a las seis especies (Fig. 2). Las secuencias genéticas 


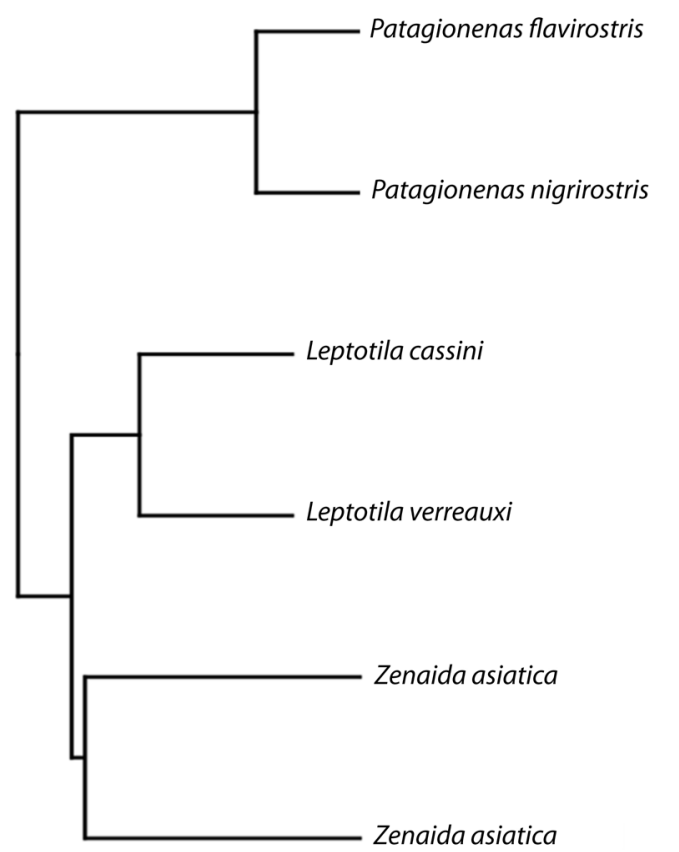

Fig. 2. Relaciones filogenéticas entre las seis especies de palomas (Columbidae) neotropicales utilizadas para relacionar la morfología alar y vuelo. El árbol de consenso filogenético fue construido utilizando la regla de la mayoría del $50 \%$ con el programa Mesquite 3.04 (Maddison \& Maddison, 2015) y derivado de 10000 árboles obtenidos de birdtree.org y generados siguiendo los métodos de construcción propuestos y explicados por Jetz, Thomas, Joy, Hartmann, \& Mooers (2012).

Fig. 2. Phylogenetic relationship between the six neotropical dove species (Columbidae) used to correlate the wing morphology and flight. The phylogenetic consensus tree was made using the $50 \%$ majority rule on Mesquite 3.04 (Maddison \& Maddison, 2015), and derived from 10000 phylogenetic trees obtained at birdtree.org, built following Jetz, Thomas, Joy, Hartmann, \& Mooers (2012).

fueron obtenidas de birdtree.org y los árboles filogenéticos fueron generados siguiendo los métodos de construcción propuestos y explicados por Jetz, Thomas, Joy, Hartmann, \& Mooers (2012). Cuando $\lambda=0$ para alguna de nuestras variables dependientes, significa que la relación entre las especies no explica los valores observados; pero si $\lambda=1$ significa que hay una relación entre la cercanía filogenética de las especies y la variable dependiente. La estimación de $\lambda$ la realizamos con BayesTraits v. 2.0 (Meade \& Pagel, 2014), usando el modelo de evolución de movimiento browniano, con el método de máxima verosimilitud. Los valores de $\lambda$ estimados se compararon contra los de $\lambda=$ 1 y $\lambda=0$ utilizando una prueba de proporción de verosimilitud que sigue una distribución chicuadrado (Meade, \& Pagel, 2014). Los valores de nuestras cinco variables respuesta no están influenciados por las relaciones filogenéticas de las especies, ya que los valores de $\operatorname{los} \lambda$ estimados difieren significativamente de $\lambda=1$ ( $\mathrm{P}$ $<0.001$ para las cinco variables).

Nuestros análisis no se realizaron separando por sexo, debido que para todas las especies analizadas el rango de traslape en medidas corporales de machos y hembras es grande (ver Baptista et al., 1997; Gibbs et al., 2001), y la mayoría de los datos se tomaron de individuos vivos que no fueron sexados (Cuadro 1). Realizamos un análisis discriminante escalonado hacia atrás e interactivo para seleccionar el menor número de variables que explicaran la mayor diferencia entre la morfología de las alas de las seis especies de palomas estudiadas (variable independiente). Comenzamos con un modelo que incluía las cinco variables (carga alar, proporción de forma, conicidad alar, y largo relativo y el área alar); luego excluimos del análisis discriminante la variable con el valor más bajo de F; después de cada exclusión el nuevo modelo fue validado utilizando el método de jackknife con 10000 permutaciones. Continuamos excluyendo las variables hasta que obtuvimos un modelo con la menor cantidad de variables que aún proporcionaban el mismo o mayor porcentaje de asignaciones correctas en relación con el modelo original que incluyó todas las medidas y estimaciones aerodinámicas del ala. Adicionalmente, realizamos una prueba binomial para probar si la clasificación obtenida por el análisis discriminante para cada especie (cuantos individuos de cada especie fueron clasificados en su propia especie) fue mayor a lo esperado por el azar (uno dividido entre seis, número de especies utilizadas). Luego realizamos un análisis de varianza de una vía para comparar como cada variable morfológica varía entre especies. Para este análisis utilizamos como factor aleatorio 
el género de cada especie (para corregir por usar múltiples especies del mismo género), donde el factor independiente fue la especie y la variable respuesta fue cada parámetro aerodinámico analizado. Al hacer un análisis por variable aerodinámica en lugar de uno solo análisis multivariable, reportamos los valores de la probabilidad ya corregidas por múltiples comparaciones. Realizamos una prueba a posteriori de Tukey para comparar los promedios de las cinco medidas alares entre las especies. Finalmente, realizamos cinco correlaciones de rangos de Spearman para comparar como varía cada una de las medidas aerodinámicas respecto a los valores del índice de vuelo utilizado para clasificar a cada especie. Los análisis estadísticos (análisis discriminante y el análisis de varianza) se realizaron utilizando el programa SYSTAT ver. 11.00.01 (SYSTAT Software, Chicago, IL, EEUU), y la correlación utilizando PAST ver. 3.21 (Hammer, Harper, \& Ryan, 2001).

\section{RESULTADOS}

En total analizamos 111 individuos adultos de ambos sexos (Cuadro 1). Encontramos que el modelo múltiple discriminante que explica mejor la separación de las seis especies de palomas analizadas utilizando la morfología alar (Wilk's $\lambda=0.08, F_{20,339}=18.69, \mathrm{P}<0.001$, Fig. 3) incluyó cuatro de las cinco variables (basada en el área alar, la proporción de forma, la conicidad alar y el largo relativo). Este modelo clasificó correctamente al $57 \%$ de los individuos dentro de su propia especie (Fig. 3).

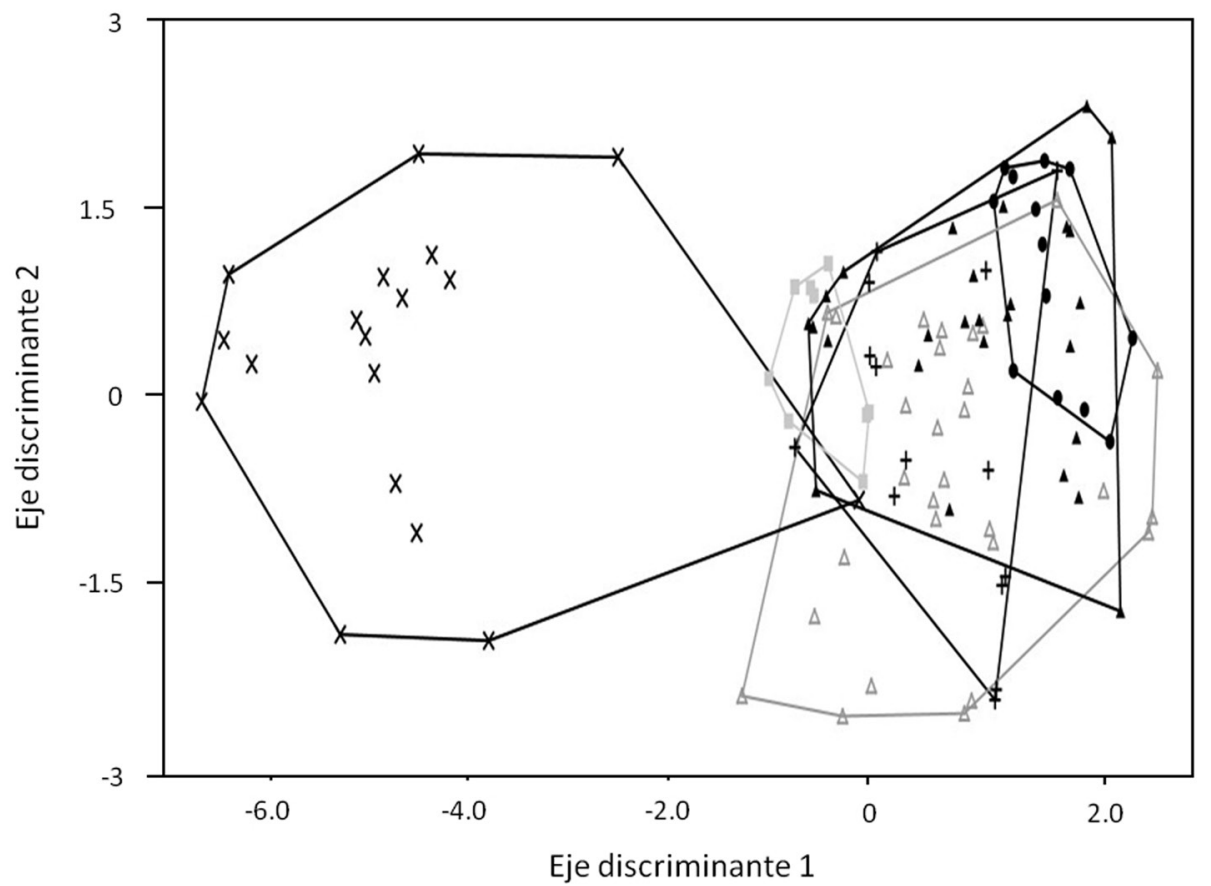

Fig. 3. Distribución de las seis especies de palomas incluidas en este estudio en el espacio definido por los ejes discriminantes 1 (99.9 \% correlacionado con el área alar) y 2 (87.6 \% correlacionado con el largo relativo y $45.0 \%$ con la proporción de forma), utilizando cuatro variables morfométricas de las alas (el área alar, la proporción de forma, la conicidad alar y el largo relativo). Equis $=$ Patagioenas flavirostris. Cuadrado gris $=P$. nigrirostris. Triángulo negro $=$ Zenaida asiatica . Triángulo gris $=$ Leptotila verreauxi . Cruz $=L$. cassini . Circulo negro $=$ Geotrygon montana .

Fig. 3. Distribution of the six dove species according to the discriminant axis 1 ( $99.9 \%$ correlated with wing area) and axis 2 ( $87.6 \%$ correlated with relative length and $45.0 \%$ with shape ratio) using four wing variables (wing area, shape ratio, wing taper, and relative length). Equis $=$ Patagioenas flavirostris. Gray square $=$ P. nigrirostris. Black triangle $=$ Zenaida asiatica . Gray triangle $=$ Leptotila verreauxi. Plus symbol $=$ L. cassini. . Black circle $=$ Geotrygon montana . 
De las 12 G. montana 11 fueron clasificadas correctamente $(92 \%)$. De las 14 L. cassini siete fueron clasificadas correctamente $(50 \%)$ y de las 30 L. verreauxi 11 fueron clasificadas correctamente (37\%); sin embargo, de los individuos clasificados erróneamente dentro de las especies de este género, dos L. cassini fueron clasificadas como $L$. verreauxi, y nueve $L$. verreauxi fueron clasificadas como $L$. cassini, indicando una similitud alta en la morfología alar entre las especies del mismo género (Fig. 3). De las 28 Z. asiatica 12 fueron clasificados correctamente (43\%). De las diez P. nigrisotris seis fueron clasificadas correctamente $(60 \%)$; y de las 17 P. flavirostris 16 fueron clasificados correctamente (94\%). Todos los porcentajes exceden el porcentaje de clasificación esperada por el azar para cada especie (prueba Binomial: $\mathrm{P}<0.01$ ).

Al comparar cada una de las variables alares entre las especies, encontramos que el área alar fue mayor en $P$. flavirostris, intermedio en $P$. nigrirostris, y menor y similar en las otras cuatro especies $\left(F_{5,105}=37.89, \mathrm{P}=0.005\right.$, Fig. 4). La mayor carga alar correspondió a $P$. flavirostris y la menor a $Z$. asiatica y $L$. verreauxi $\left(F_{5,105}=4.12, \mathrm{P}=0.01\right.$, Fig. 4$)$. Tanto $L$. verreauxi como $L$. cassini tuvieron las alas proporcionalmente más anchas o cuadradas (con valores menores de proporción de forma) en comparación con las otras cuatro especies, las cuales presentaron alas con la proporción de forma similar entre ellos $\left(F_{5,105}=5.67, \mathrm{P}<\right.$ 0.005 , Fig. 4). Z. asiatica tuvo alas más puntiagudas, mientras que $G$. montana presentó alas con una punta más redondeada $\left(F_{5,105}=4.46, \mathrm{P}\right.$ $=0.005$, Fig. 4). El largo alar relativo fue mayor en Z. asiatica que en las otras cinco especies de palomas, en las cuales el largo alar fue menor y similar entre ellas $\left(F_{5,105}=3.78, \mathrm{P}=0.015\right.$, Fig. 4). Encontramos que a mayor índice de vuelo (Fig. 5) las especies presentaron alas con áreas mayores $\left(\mathrm{r}_{\mathrm{s}}=0.57, \mathrm{P}<0.001\right)$, una proporción de forma más rectangular $\left(\mathrm{r}_{\mathrm{s}}=0.27, P\right.$ $=0.005)$, más puntiagudas $\left(\mathrm{r}_{\mathrm{s}}=0.23, \mathrm{P}=0.23\right)$, y un largo relativo mayor $\left(r_{s}=0.39, P<0.001\right)$. Pero la carga alar fue similar entre especies $\left(\mathrm{r}_{\mathrm{s}}\right.$ $=0.10, \mathrm{P}=0.29$, Fig. 5).

\section{DISCUSIÓN}

La familia Columbidae (palomas y afines) presenta un modelo corporal muy conservado y por lo tanto una morfología poco variable y fácilmente identificable (Gibbs et al., 2001). Sin embargo, dentro de esta familia de aves hay una gran variedad de estrategias de vuelo. Por ejemplo, en especies de los géneros Geotrygon, Gallicolumba y Goura los individuos se desplazan casi exclusivamente caminando; mientras que en otros géneros como Columba, Patagioenas y Ducula se desplazan casi exclusivamente volando (Skutch, 1991; Gibbs et al., 2001). Sin embargo, esta clasificación no es discreta, ya que hay muchas otras especies (ej., Columbina, Caloenas, Leptotila o Zenaida) que combinan en diferente proporción el tiempo invertido en desplazarse volando con caminar sobre el suelo, creando un continuo entre ambos modos de desplazamiento (Skutch, 1991; Gibbs et al., 2001). Nuestros resultados muestran que la morfología alar (utilizando únicamente cuatro variables) permite una separación, de media a alta, de las seis especies de palomas analizadas (P. flavirostris, $P$. nigrirostris, $Z$. asiatica, $L$. verreauxi, $L$. cassini y $G$. montana). Estos resultados indican que las diferencias en la forma de las alas pueden estar asociadas con el tiempo que invierte cada especie para desplazarse volando o caminando, ya que las variables que permiten la separación fueron el área alar, la proporción de forma, la conicidad alar y el largo relativo, todas asociadas con diferentes tipos de vuelo (Fig. 3).

Las alas más cónicas y con una proporción de forma más rectangular o alargada de $P$. flavirostris, y en menor medida la de $P$. nigrisortris, resulta en una forma más eficiente para vuelos extensos y por lo tanto asociada a los hábitats abiertos que utilizan estas especies para desplazarse y alimentarse, como el dosel del bosque o las copas de los árboles en zonas abiertas (Stiles, \& Skutch, 1989; Gibbs et al., 2001; Lapiedra et al., 2013). Este tipo de morfología alar está presente en otras aves que son voladoras activas de zonas abiertas, donde se necesita poca maniobrabilidad y vuelos 

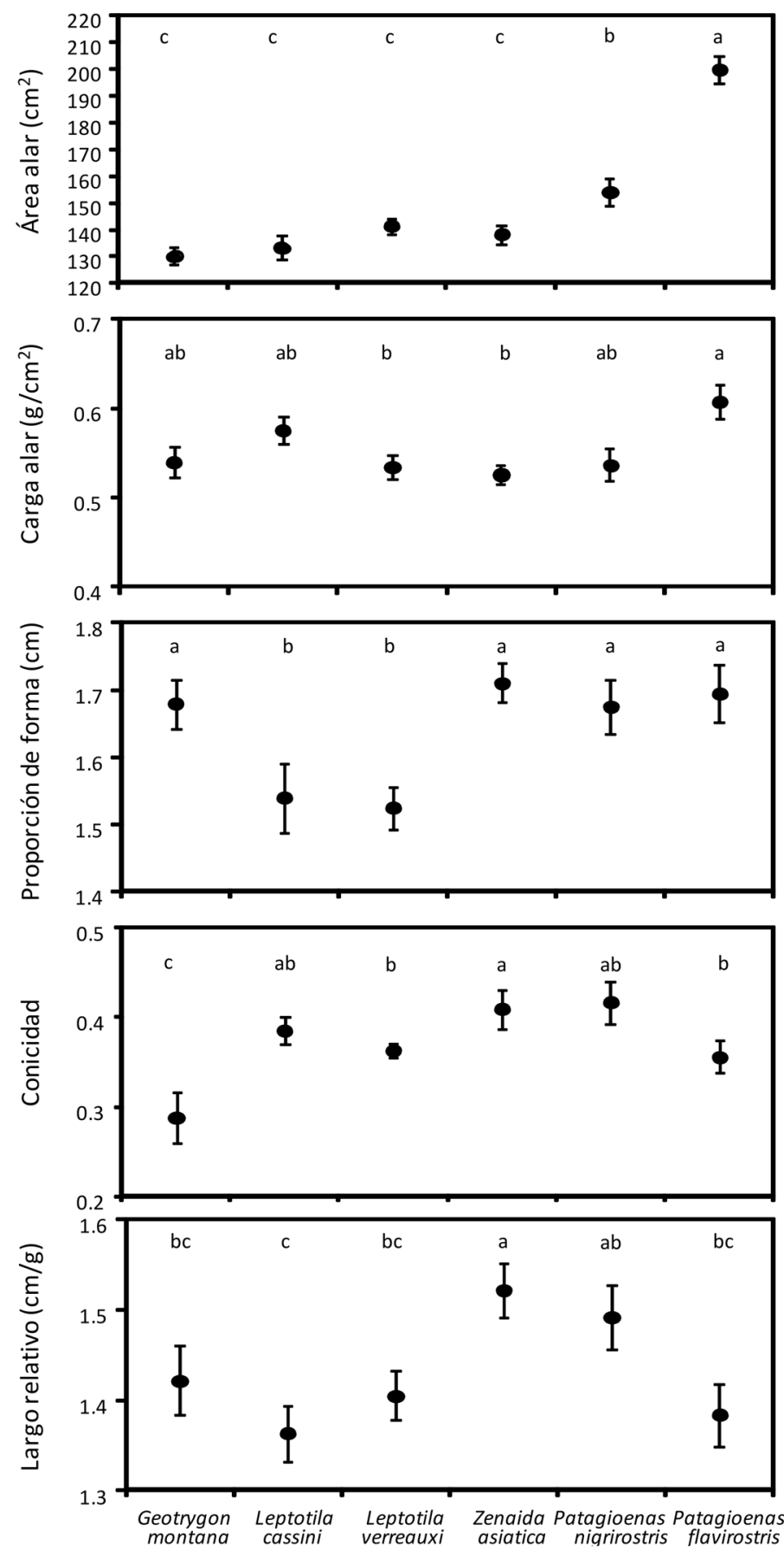

Fig. 4. Promedio ( \pm EE) de cinco variables morfométricas alares de las seis especies de palomas incluidas en este estudio. Especies con la misma letra no son significativamente diferentes según la prueba a posteriori de Tukey.

Fig. 4. Mean $( \pm \mathrm{SE})$ of the five morphometric variables for the six dove species used in this study. Species with the same letter on top are not significantly different according to the Tukey post hoc test. 

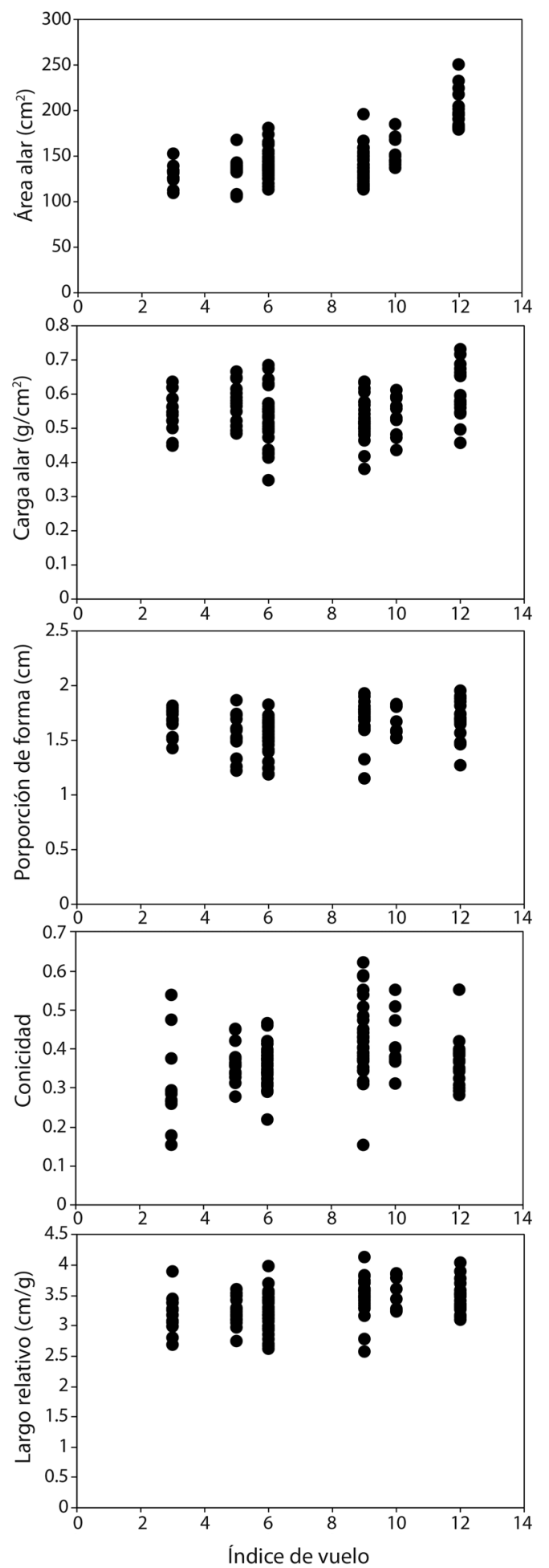

energéticamente más eficientes (Savile, 1957; Norberg, 1985).

La morfología alar de G. montana está más adaptada a los hábitats densos de los sotobosques, donde es común encontrar a esta especie desplazándose (caminando sobre el suelo) y alimentándose (Stiles, \& Skutch, 1989; Skutch, 1991; Gibbs et al., 2001). Las alas de G. montana tienen puntas redondeadas y una proporción de forma que tiende a ser cuadrada. Previamente se ha demostrado que este tipo de morfología alar maximiza la aceleración en vuelos explosivos y permite cambios rápidos de dirección (mayor maniobrabilidad) (Kirmse, 1998; Lockwood et al., 1998), características que favorecen huir de depredadores de acecho en ambientes densos, donde los espacios abiertos son limitados.

Las especies de los géneros Zenaida y Leptotila tienen morfologías alares intermedias (una proporción de forma cuadrada, alas puntiagudas y baja carga alar) entre las especies de Patagioenas y Geotrygon, y esto está asociado a que las especies en ambos géneros utilizan una mezcla de vuelo y caminata para desplazarse (Stiles, \& Skutch, 1989; Skutch, 1991; Gibbs et al., 2001). Además, ambos géneros utilizan hábitats que incluyen una

Fig. 5. Correlaciones de rangos de Spearman entre el índice de vuelo (ver métodos para una explicación de su estimación) para cada especie de paloma y las cinco variables morfométricas alares. Valores bajos representan poca capacidad de vuelo, habitar vegetación densa y cerca del suelo. Valores altos representan alta capacidad de vuelo, vivir en hábitats abiertos y en el dosel. Valores para cada especie: Geotrygon montana $=3$, Leptotila cassini $=5$, L. verreauxi $=6$, Zenaida asiatica $=9$, Patagioenas nigrirostris $=10, P$. flavirostris $=12$.

Fig. 5. Spearman rank correlations between flight index (see methods for understand how is estimated) for each dove species and the five morphometric wing variables. Low values mean low flight capacity and inhabit dense vegetation close to the ground. High values mean high flight capacity and inhabit in the canopy and open areas. Values for each species: Geotrygon montana $=3$, Leptotila cassini $=5, L$. verreauxi $=6$, Zenaida asiatica $=9$, Patagioenas nigrirostris $=10, P$. flavirostris $=12$. 
mezcla de parches densos de vegetación y zonas abiertas para alimentarse, reproducirse y refugiarse (Stiles, \& Skutch, 1989; Skutch, 1991; Gibbs et al., 2001), por lo que alas con características intermedias permiten maniobrar eficientemente dentro de ambientes densos, pero a la vez mantener vuelos prolongados y energéticamente eficientes en áreas abiertas (Videler, 2005). Entre estos dos géneros, la mayor conicidad alar y el ala alargada en $Z$. asiatica puede deberse a que presenta una mayor proporción de vuelo que las especies en el género Leptotila.

Las especies de Patagioenas, Leptotila y Geotrygon presentaron valores altos de carga alar y similares entre sí, esto se puede deber a que las especies de Patagioenas presentan un mayor peso corporal que las especies en los otros tres géneros (Stiles, \& Skutch, 1989; Gibbs et al., 2001), lo que aumenta la carga alar. En el caso de las especies de Leptotila y G. montana la capacidad de carga alar elevada puede ser el resultado de alas cortas y redondeadas como una adaptación en estas aves para producir vuelos cortos y explosivos que les permita escapar o eludir rápidamente el ataque de algún depredador, como ocurre en otros grupos de aves (Schulenberg, 1983; Sandoval, 2009).

En conclusión, encontramos que el tipo de desplazamiento predominante y uso de hábitat en cada una de las seis especies de Columbidae analizadas, influye directamente en la morfología alar, aún entre especies cercanamente emparentadas. Otros factores, además de los estudiados aquí, podrían influir en la forma del ala, pero el uso del hábitat y tipo de desplazamiento explica un alto porcentaje de la variación en forma. Por ejemplo, las especies $P$. flavirostris y $P$. nigrirostris tiene alas adaptadas para vuelos en zonas abiertas, en $G$. montana alas adaptadas para vuelos explosivos y maniobrables en hábitats densos, y alas con características intermedias en las especies $L$. verreauxi L. cassini y $Z$. asiatica.

Declaración de ética: los autores declaran que todos están de acuerdo con esta publicación y que han hecho aportes que justifican su autoría; que no hay conflicto de interés de ningún tipo; y que han cumplido con todos los requisitos y procedimientos éticos y legales pertinentes. Todas las fuentes de financiamiento se detallan plena y claramente en la sección de agradecimientos. El respectivo documento legal firmado se encuentra en los archivos de la revista.

\section{AGRADECIMIENTOS}

Queremos dar gracias a los curadores del Museo Nacional de Costa Rica por brindarnos acceso a los especímenes depositados en la colección de aves. También agradecemos a tres revisores anónimos por sus valiosos comentarios en una versión previa del manuscrito. LS agradece a Vicerrectoría de investigación por el apoyo mediante el proyecto de investigación Colecciones del Museo de Zoología 11-B6773, y a la Escuela de Biología por el nombramiento de medio tiempo.

\section{RESUMEN}

Los factores biomecánicos, el comportamiento e historia natural de las especies pueden influenciar la forma y dimensión del ala en las aves. Por lo tanto, la morfología de las alas de cada especie podría estar adaptada al ambiente en el que habitan. En palomas (familia Columbidae) existe un gradiente de especies que tienden a volar mucho y otras que vuelan muy poco. El objetivo de esta investigación es determinar si la forma de las alas de seis especies de palomas se asocia con su capacidad de vuelo. Predecimos que las especies Patagioenas flavirostris y P. nigrirostris que tienden a volar largas distancias presentan alas relativamente largas y puntiagudas; mientras que Geotrygon montana que se desplaza principalmente caminando presenta alas relativamente anchas y redondeadas. Especies con capacidad de vuelo intermedio (Leptotila verreauxi, $L$. cassini y Zenaida asiatica) van a presentar alas con características intermedias. Estimamos la carga alar, proporción de la forma, conicidad alar, largo relativo y el área alar promedio para cada especie. Realizamos un análisis discriminante para determinar cuál combinación de variables explica mejor la diferencia morfológica de las alas entre las seis especies y probamos que tan robusto es este modelo mediante una prueba binomial. El modelo clasificó correctamente el $57 \%$ de los individuos en su propia especie. Por lo tanto, encontramos que el tipo de desplazamiento predominante en cada una de las seis especies de Columbidae 
analizadas, se asocia con la morfología alar: alas adaptadas para vuelos en zonas abiertas en $P$. flavirostris y P. nigrirostris, alas adaptadas para vuelos muy maniobrables en hábitats densos en G. montana, y alas con características intermedias en $L$. verreauxi, L. cassini y Z. asiatica.

Palabras clave: Parámetros aerodinámicos; comportamiento de vuelo; Efecto de Hábitat; Palomas; forma del ala.

\section{REFERENCIAS}

Arizaga, J., Campos, F., \& Alonso, D. (2006). Variation in wing morphology among subspecies might reflect different migration distances in Bluethroated. Ornis Fennica, 83, 162-169.

Baptista, L. F., Trail, P. W., \& Horblit, H. M. (1997). Pigeons, Doves (Columbidae). In J. del Hoyo, A. Elliott, J. Sargatal, D. A. Christie, \& E. de Juana (Eds.), Handbook of the Birds of the World Volume 4: Sandgrouse to Cuckoos (pp. 60-243). Barcelona, Spain: Lynx Edicions.

Corvidae, E. L., Bierregaard, R. O., \& Peters, S. E. (2006). Comparison of wing morphology in three birds of prey: correlations with differences in flight behavior. Journal of Morphology, 267, 612-622. DOI: 10.1002/ jmor. 10425

Dial, K. P. (1992). Avian forelimb muscles and nonsteady flight: can birds fly without using the muscles in their wings?. Auk, 109, 874-885. DOI: 10.2307/4088162

Gibbs, D., Barnes, E., \& Cox, J. (2001). Pigeons and Doves. A Guide to the Pigeons and Doves of the World. New Haven, Connecticut, USA: Yale University Press.

Hammer, Ø., Harper, D. A. T., \& Ryan, P. D. (2001). PAST: Paleontological statistics software package for education and data analysis. Palaeontologia Electronica, 4(1),1-9. Recuperado de http://palaeo-electronica. org/2001_1/past/issue1_01.htm

Jetz, W., Thomas, G. H., Joy, J. B., Hartmann, K., \& Mooers, A. O. (2012). The global diversity of birds in space and time. Nature, 491, 444-448. DOI: 10.1038/ nature 11631

Kirmse, W. (1998). Morphometric features characterizing flight properties of palearctic eagles. In R. D. Chancellor, B. U. Meyburg, \& J. J. Ferrero (Eds.), Holarctic birds of prey (pp. 339-348). Berlin, Alemania: Proceedings of an international conference.

Lapiedra, O., Sol, D., Carranza, S., \& Beaulieu, J. M. (2013). Behavioural changes and the adaptive diversification of pigeons and doves. Proceedings of the Royal Society of London B, 280, 20122893. DOI: $10.1098 / \mathrm{rspb} .2012 .2893$

Lockwood, R., Swaddle, J. P., \& Rayner, J. M. (1998). Avian wingtip shape reconsidered: wingtip shape indices and morphological adaptations to migration. Journal of Avian Biology, 29, 273-292. DOI: $10.2307 / 3677110$

Maddison, W. P., \& Maddison, D. R. (2015). Mesquite: a modular system for evolutionary analysis (Version 3.04). Recuperado de http://mesquiteproject.org

Meade, A., \& Pagel, M. (2014). BayesTraits (Version 2.0). Recuperado de http://www.evolution.rdg.ac.uk/ BayesTraits.html

Norberg, U. M. (1981). Flight, morphology and the ecological niche in some birds and bats. Symposia of the Zoological Society of London, 48, 173-197.

Norberg, U. M. (1985). Flying, gliding, and soaring. In M. Hildebrand, D. M. Bramble, K. F. Liem, \& D. B. Wake (Eds.), Functional vertebrate morphology (Pp. 129-158). Cambridge, Massachusetts, USA: Harvard University Press.

Norberg, U. M. (1995). How a long tail and changes in mass and wing shape affect the cost for flight in animals. Functional Ecology, 9, 48-54. DOI: $10.2307 / 2390089$

Pennycuick, C. J. (2008). Modelling the flying bird (Vol. 5). USA: Elsevier.

Sandoval, L. (2009). Descripción del comportamiento de cuido parental en la codorniz de monte Colinus leucopogon. International Journal of Galliformes Conservation, 1, 36-40.

Savile, D. B. (1957). Adaptive evolution in the avian wing. Evolution, 11, 212-224.

Schneider, C. A., Rasband, W. S., \& Eliceiri, K. W. (2012). NIH Image to ImageJ: 25 years of image analysis. Nature Methods, 9, 671-675. DOI: 10.1038/ nmeth.2089

Schulenberg, T. S. (1983). Foraging behavior, eco-morphology, and systematics of some antshrikes. Wilson Bulletin, 95, 505-521.

Skutch, A. F. (1991). The life of the pigeon. Ithaca, New York, USA: Comstock Publishing.

Stiles, F. G., Altshuler, D. L., \& Dudley, R. (2005). Wing morphology and flight behavior of some North American hummingbird species. Auk, 122, 872-886. DOI: $10.1642 / 0004-8038(2005) 122[0872$ :WMAFB O]2.0.CO;2

Stiles, G. F., \& Skutch, A. F. (1989). A guide to the birds of Costa Rica. Ithaca, New York, USA: Cornell University Press.

Videler, J. J. (2005). Avian flight. Oxford, UK: Oxford University Press. 